\title{
Experimental Investigation of a Prediction Algorithm for an Indoor SLAM Platform*
}

\author{
Jung-Fu Hou ${ }^{1}$, Yu-Shin $\mathrm{Chou}^{2}$, Yau-Zen Chang ${ }^{1}$, and Jing-Sin Liu ${ }^{2, *}$ \\ ${ }^{1}$ Department of Mechanical Engineering, Chung-Gung University, Taiwan \\ ${ }^{2}$ Institute of Information Science, Academia Sinica, Taiwan \\ liu@iis.sinica.edu.tw
}

\begin{abstract}
This paper presents a scheme for the indoor simultaneous localization and mapping (SLAM) problem. The scheme is based on the scan matching method and is treated as an optimization problem solve by the Simplex method. The two-dimensional distance transform method is used to facilitate the cost value evaluation. In order to register scanned maps with built map through maximum overlap between the maps, a predictive algorithm is proposed. The algorithm can not only reduce search scope but also discard unexpected objects that may cause false match. The approach is investigated by an experimental platform with differential drives. The ICP-SLAM is also implemented for performance comparison. Experimental result shows that the prediction algorithm can improve accumulation error in the indoor environment.
\end{abstract}

Keywords: Simultaneous localization and mapping, Simplex method, Iterative closest point.

\section{Introduction}

In order to make mobile robot to have intelligent behavior such as path planning, obstacles avoiding. Mobile robot needs to know its position within environments. Self localization and map construct is an importance issue and need to be solved. This problem also calls a simultaneous localization and mapping (SLAM) [1][2][3][4]. Two-dimensional laser range finder (LRF) is one of the common used sensors to solve this problem. In laser scan matching method, the position and the orientation of the vehicle is estimated by rotating and translating the current scanned map and compare to the reference map until the max overlap is achieved. In modern SLAM approaches, some research use extended Kalman filter (EKF) to combine the information from laser scan and odometry data to solve SLAM problem. This form of SLAM is known as EKF-SLAM [5][6]. The main problem is laser scanned data need to be analyzed and to form a set of feature points. These feature point usually come from the geometry properties of scanned data such as corner, line curvature. Due to the lack of feature point detection, this may cause lower system performance and inaccuracy map mapping. Another kind of approach is used scan matching method. This method can be categorized into feature to feature, point to feature, point to point. In the

\footnotetext{
${ }^{*}$ Corresponding author.
} 
feature to feature approaches, the property of laser scanned data, such as line segments, corners are extracted then matched directly [7]. This kind of approach requires the extract features from laser scanned data precisely. In the point to feature approaches, the feature such as line is extracted from reference scanned data and forms a predefined map. Then current scanned data is matched to this predefined map. The point to point approaches do not need to analysis scanned data. The iterative closest point (ICP) is the most popular method to align two different point set [8][9]. In this method, the smallest Euclidean distance for every point in current scanned data to the reference scanned data is calculated to be the cost value to estimate the best overlap. The ICP method has better convergence performance in translation then rotation. The IMRP method [9][10] is then proposed to estimate the rotation.

In this paper, we present a scheme to solve SLAM problem. This scheme treat mapping problem as an optimization problem. In order to achieve the fast convergence, Simplex method is used and Occupy grid map method is used to reduce the size of computation data. Unlike the ICP or IMPR, Euclidean distances are precalculated and form a distance map before optimization process. A predict algorithm is used to find out the maximum overlap in two different maps.

The rest paper is organized as follows: distance transform is described in section 2.1. The concept of prediction algorithms is described in section 2.2. The Simplex method is described in section 2.3. Then the experiment is in section 3. Finally the conclusion is shown in section 4 .

\section{Method}

\subsection{Distance Transform}

The concept of distance transform (DT) is to calculate the Euclidean distance from certain position on map to the closest obstacle. The example of DT is shown in Fig. 1. Before distance transform, the value of obstacle position is set to zero and other position is set to infinity (Fig1. (a) ). After distance transform, the distance of every nonobstacle position to the closest obstacle is set, as shown in Fig 1. (b).

\begin{tabular}{|l|l|l|l|l|l|l|l|l|l|}
\hline Inf & Inf & Inf & Inf & Inf & Inf & Inf & Inf & Inf & Inf \\
\hline Inf & Inf & Inf & Inf & Inf & Inf & Inf & Inf & Inf & Inf \\
\hline Inf & Inf & 0 & Inf & Inf & Inf & 0 & Inf & Inf & Inf \\
\hline Inf & Inf & 0 & Inf & Inf & Inf & 0 & Inf & Inf & Inf \\
\hline Inf & Inf & 0 & Inf & Inf & Inf & 0 & Inf & Inf & Inf \\
\hline Inf & Inf & 0 & Inf & Inf & Inf & 0 & Inf & Inf & Inf \\
\hline Inf & Inf & 0 & 0 & 0 & 0 & 0 & Inf & Inf & Inf \\
\hline Inf & Inf & Inf & Inf & Inf & Inf & Inf & Inf & Inf & Inf \\
\hline Inf & Inf & Inf & Inf & Inf & Inf & Inf & Inf & Inf & Inf \\
\hline Inf & Inf & Inf & Inf & Inf & Inf & Inf & Inf & Inf & Inf \\
\hline
\end{tabular}

(a)

Fig. 1. (a) map before distance transform. (b) result of distance transform 


\begin{tabular}{|l|l|l|l|l|l|l|l|l|l|}
\hline 4 & 3 & 2 & 3 & 4 & 3 & 2 & 3 & 3 & 4 \\
\hline 3 & 2 & 1 & 2 & 3 & 2 & 1 & 2 & 2 & 3 \\
\hline 2 & 1 & 0 & 1 & 2 & 1 & 0 & 1 & 2 & 3 \\
\hline 2 & 1 & 0 & 1 & 2 & 1 & 0 & 1 & 2 & 3 \\
\hline 2 & 1 & 0 & 1 & 2 & 1 & 0 & 1 & 2 & 3 \\
\hline 2 & 1 & 0 & 1 & 1 & 1 & 0 & 1 & 2 & 3 \\
\hline 2 & 1 & 0 & 0 & 0 & 0 & 0 & 1 & 2 & 3 \\
\hline 3 & 2 & 1 & 1 & 1 & 1 & 1 & 2 & 2 & 3 \\
\hline 4 & 3 & 2 & 2 & 2 & 2 & 2 & 2 & 3 & 4 \\
\hline 5 & 4 & 3 & 3 & 3 & 3 & 3 & 3 & 3 & 4 \\
\hline
\end{tabular}

(b)

Fig. 1. (continued)

In this paper, data from laser range finder is formatted as the angle $(\phi)$ and relate distance (d) can be denoted as:

$$
D=\left[\begin{array}{lll}
\phi_{1} & \phi_{2} & \phi_{3} \ldots \phi_{n} \\
d_{1} & d_{2} & d_{3} \ldots d_{n}
\end{array}\right]
$$

The $D$ are then transform into Cartesian coordinate and expressed as:

$$
D_{c}=\left[\begin{array}{ccc}
x_{1} & x_{2} & x_{3} \ldots x_{n} \\
y_{1} & y_{2} & y_{3} \ldots y_{n}
\end{array}\right]
$$

According to $D_{c}$, an obstacle map can be built, as shown in Fig. 2 .

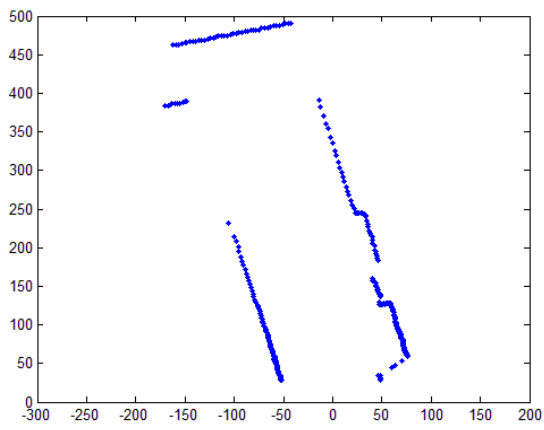

Fig. 2. Obstacle Map

Then the distance transform are then applied on obstacle map to form a DT Map. If we take the value of distance as Z-Axis, a three-dimensional figure can be form as shown in Fig. 3. In this figure, the darkest color (blue) indicates the position on the 


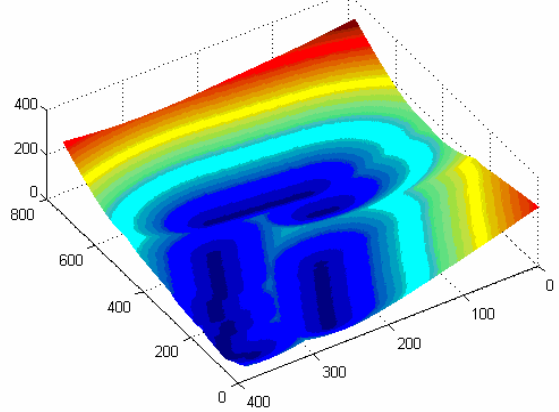

Fig. 3. The result of distance transform

map is closer than the bright color (red). This map will be used to generate cost value in the optimization process.

\subsection{Map Prediction}

For the two different time step scanned map $(P(t), P(t+1))$, maps will change due to the motion of vehicle. Compare to these two maps, some points will disappear and some point will be discovered. These points will affect the mapping result. In order to find out the max overlap area, a prediction algorithm is proposed. According to the odometry of vehicle, a roughly position of vehicle can be estimated. By using this information, a virtual laser scanned area can be applied to predict the overlap points. The concept of map prediction is shown in Fig. 4; the rectangle indicated the virtual scanned area. The figure 4(a) shows the predict area from $P(t)$ to $P(t+1)$. And the Fig.4(b) indicated the predict area from $P(t+1)$ back to $P(t)$ in the forward motion.

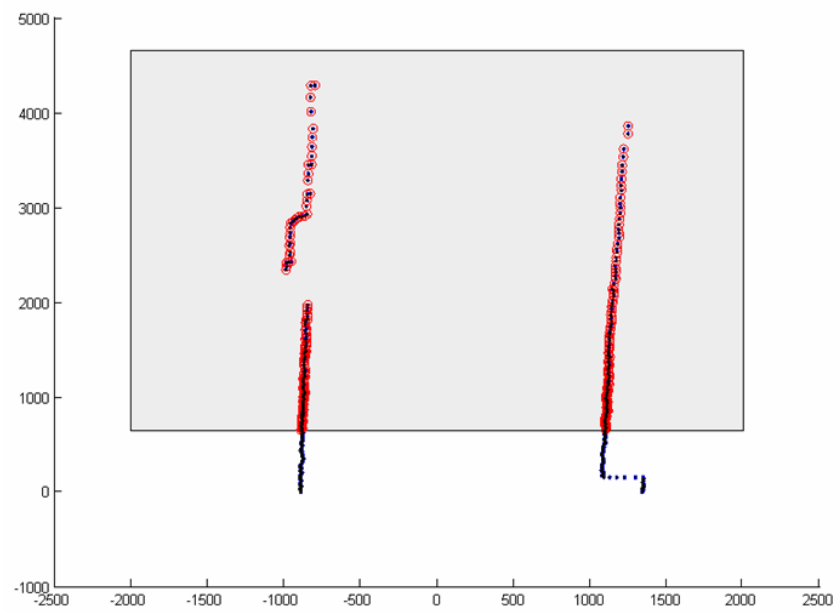

(a)

Fig. 4. (a) Prediction $P(t)$ to $P(t+1)$. (b) Prediction $P(t+1)$ back to $P(t)$ 


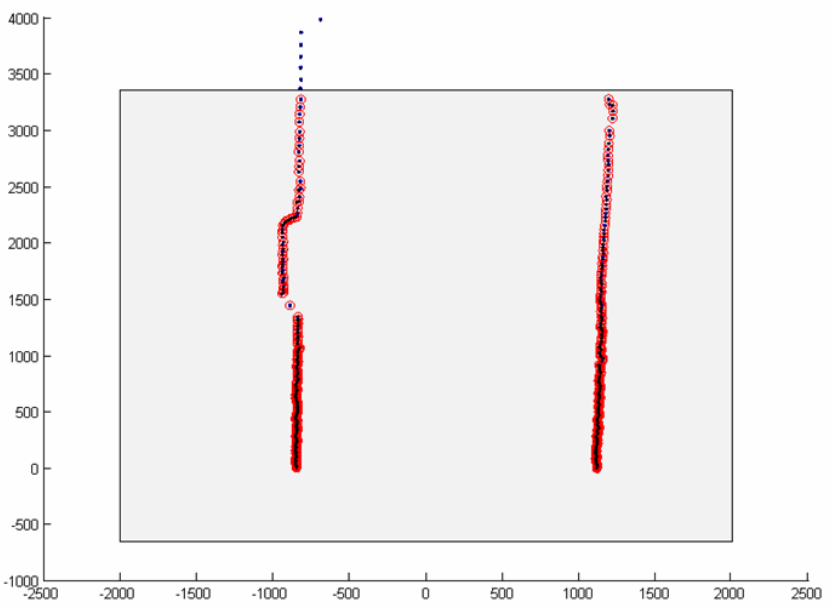

(b)

Fig. 4. (continued)

In the experiment, the motion vehicle is separated into two modes: forward and rotation mode. The pseudo code is shown below:

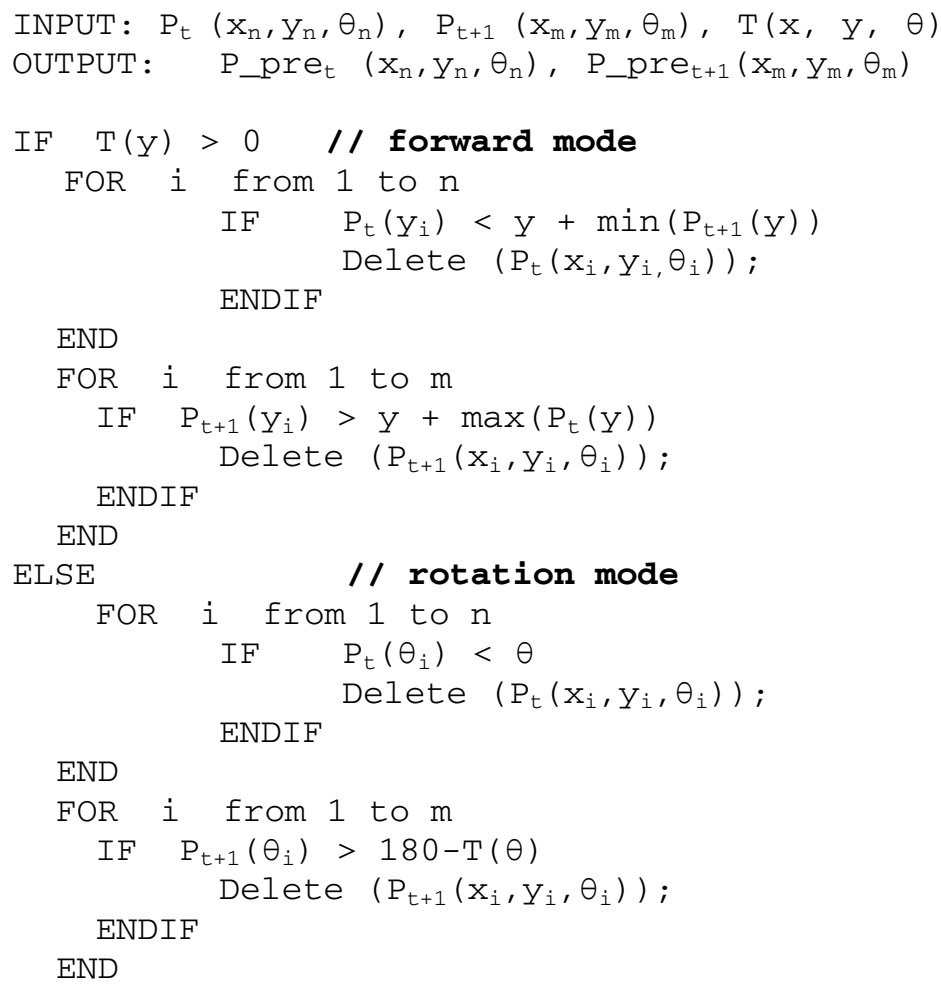




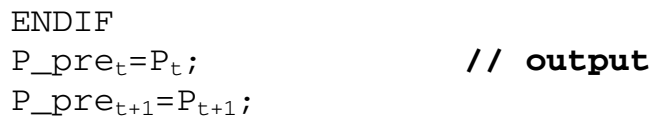

From the above method, Fig. 5 shows two maps before and after prediction in rotation mode. The differences between two maps are shown in the green circle in Fig. 5(a) and (b). After the prediction process, the point that will disappear in next time step is discarded. And max overlap area of two maps is achieved to perform mapping.

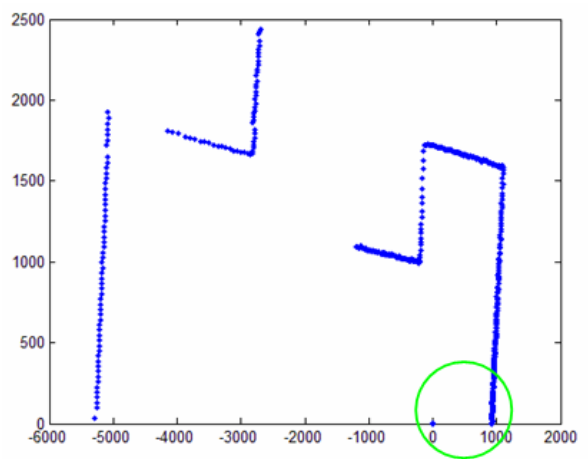

(a)

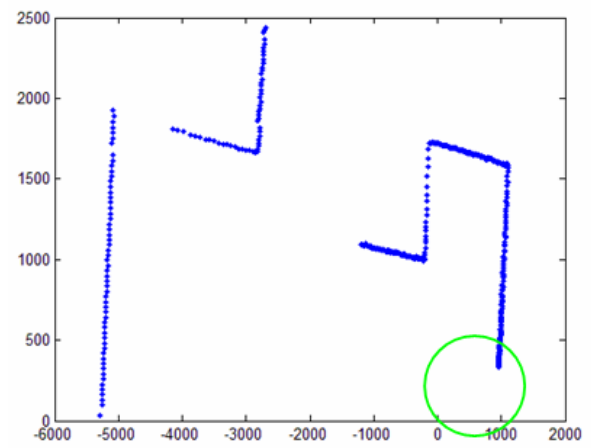

(b)

Fig. 5. (a) Map before prediction. (b) map after prediction.

\subsection{Simplex Method in Map Mapping}

Simplex method is a kind of optimization algorithm to estimate the optimal value in the cost function. The purpose of simplex method is to estimate the relation between two different maps $(P(t)$ and $P(t+1))$. This relation can define by a transformation 
matrix as below. Where $\mathrm{x}, \mathrm{y}, \theta$ are defined as the $\mathrm{x}$-axis, $\mathrm{y}$-axis shift and rotation angle separately.

$$
{ }_{t+1}^{t} T=T(x, y, \theta)=\left[\begin{array}{cccc}
\cos \theta & -\sin \theta & 0 & x \\
\sin \theta & \cos \theta & 0 & y \\
0 & 0 & 0 & 0 \\
0 & 0 & 0 & 1
\end{array}\right]
$$

For different time step maps such as $P(t+n)$ can be simply multiply on transformation estimated before, as $\mathrm{Eq}(4)$.

$$
{ }_{t+n}^{t} T={ }_{t+1}^{t} T \cdot{ }_{t+2}^{t+1} T \ldots{ }^{t+(n-1)} T={ }_{t+n}{ }_{t+(n-1)}^{t} T \cdot{ }^{t+(n-1)} T
$$

In the definition of cost function, after distance transform is applied on reference map $(P(t))$. The prediction algorithms are applied on current scanned point data $(P(t+1))$ to discard unexpected point. And then transfer to reference to reference DT map according following equation.

$$
P_{\text {trnas }}={ }_{t+1}^{t} T \cdot P_{\text {current }}
$$

Then the cost value is defined as fig. 6. The red color grid on the distance map denoted the obstacle point on the reference map $(P(t))$. And the yellow color grid is the transformed point data project on to the distance map, and the sum of distance $\left(D_{\text {sum }}\right)$ of yellow grid is used as cost value. The Simplex method is used to estimate the smallest $D_{\text {sum }}$.

\begin{tabular}{|c|c|c|c|c|c|c|c|c|c|}
\hline 22 & 14 & 10 & 14 & 22 & 28 & 36 & 42 & 50 & 57 \\
\hline 20 & 10 & 0 & 10 & 14 & 22 & 28 & 36 & 42 & 50 \\
\hline 22 & 14 & 10 & 0 & 10 & 14 & 22 & 28 & 36 & 42 \\
\hline 28 & 22 & 14 & 10 & 0 & 10 & 14 & 22 & 28 & 36 \\
\hline 36 & 28 & 22 & 14 & 10 & 0 & 10 & 14 & 22 & 32 \\
\hline 42 & 36 & 28 & 22 & 14 & 10 & 0 & 10 & 20 & 30 \\
\hline 36 & 28 & 22 & 14 & 10 & 0 & 10 & 14 & 22 & 32 \\
\hline 32 & 22 & 14 & 10 & 0 & 10 & 14 & 22 & 28 & 36 \\
\hline 30 & 20 & 10 & 0 & 10 & 14 & 22 & 28 & 36 & 42 \\
\hline 32 & 22 & 14 & 10 & 14 & 22 & 28 & 36 & 42 & 50 \\
\hline
\end{tabular}

Fig. 6. Example of cost calculation 
The complete flowchart of proposed scheme is shown in Fig. 7.

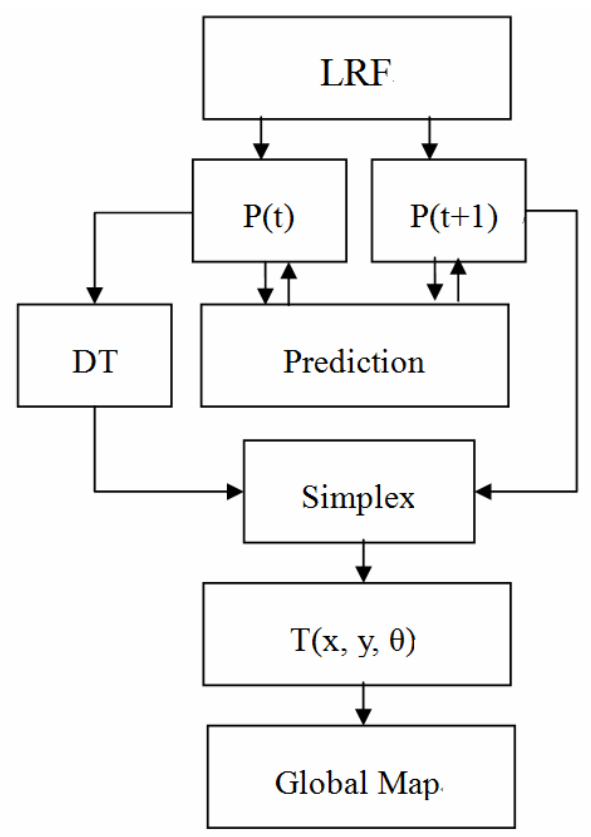

Fig. 7. Flow chart of proposed scheme

\section{Experiment}

In this paper, a vehicle with differential drive is used, as shown in Fig. 8. Laser range finder is mounted in the front of vehicle. A notebook is used to control the vehicle and perform the SLAM task. Two motor made by MONTROL is used and controlled through RS-232. The laser range finder is manufactured by HOKUYO URG-04LX. Scanned data is captured through USB.

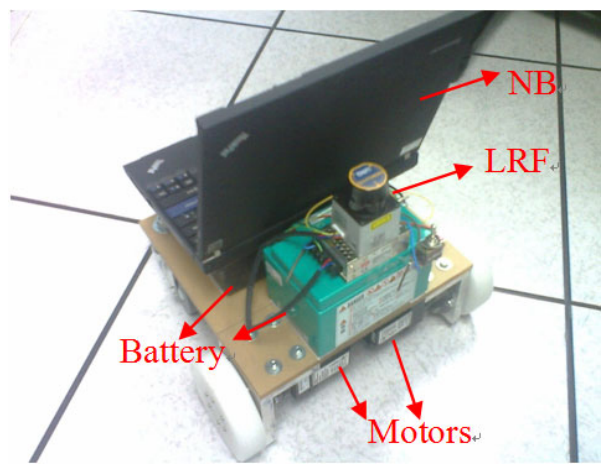

Fig. 8. Picture of experimental vehicle 
The control structure of mobile robot is shown in Fig. 9. The robot is driven by two motor. Data from LRF is received by a notebook through RS-232, Sample rate is 2 seconds. The direction of robot move is controlled by a joystick.

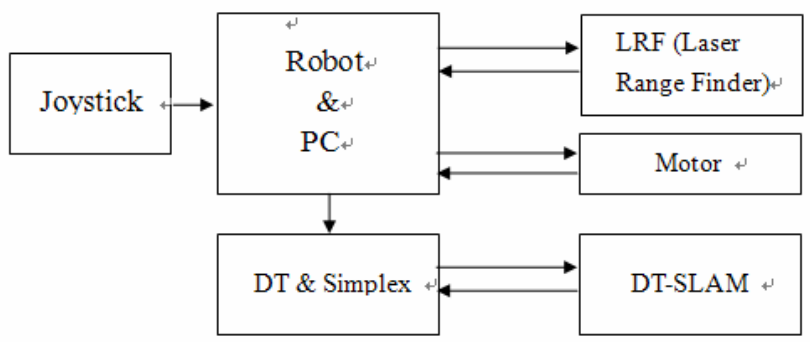

Fig. 9. Control structure of mobile robot

In the experiment, two methods are implemented to validate the performance of proposed scheme: odometry only, and ICP-SLAM. The experiment is in a $30 \mathrm{~m} \times 5 \mathrm{~m}$ indoor environment. In the first experiment, we drive vehicle to move around rectangular like environment. The robot is driven to move around this area to observe the accumulation error of proposed scheme. The result of experiment is shown in Figure 10.
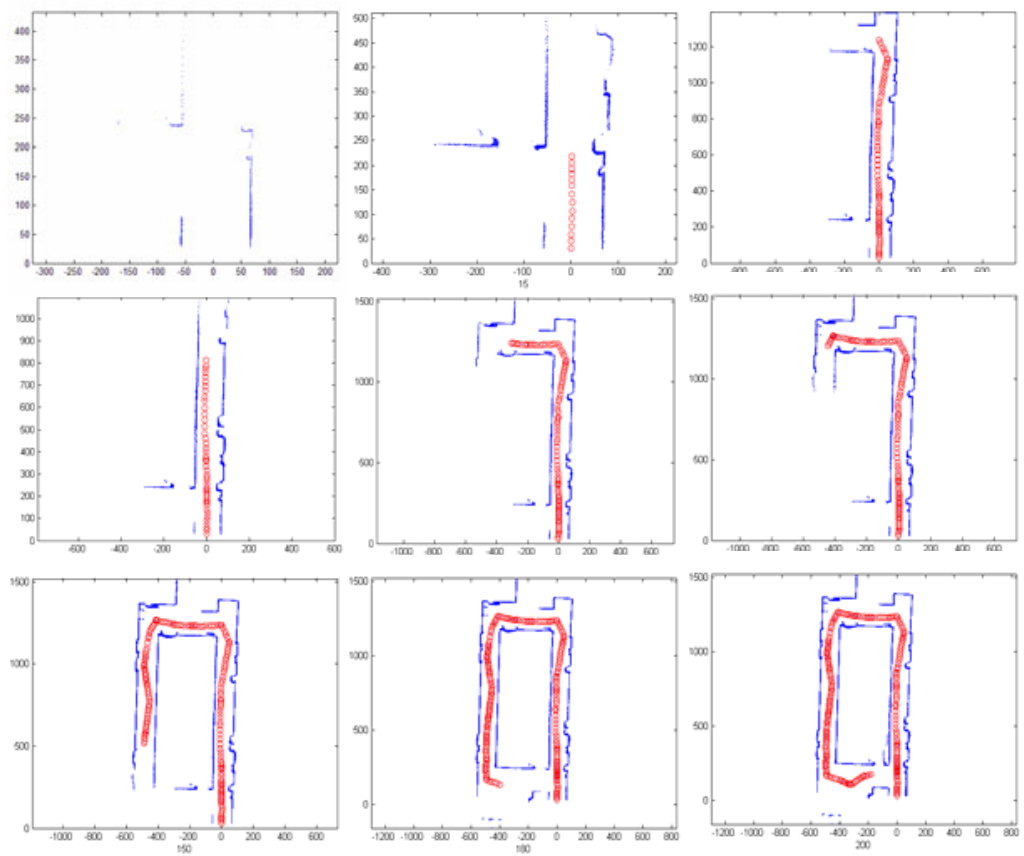

Fig. 10. Result for test environment 
The experimental result of ICP and odometry only method is shown in Fig. 11. Compared with the ICP method, serious accumulation errors problem can be observed. These errors cause the SLAM map inaccuracy. The odometry only method also can find such problems.
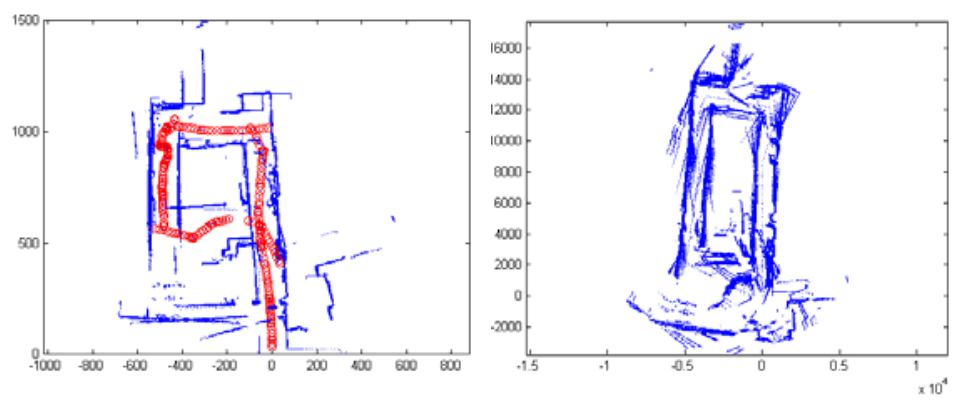

Fig. 11. (a) Result of ICP method (b) Result of odometry only

In order to test the performance of the prediction algorithm, the same scheme except prediction algorithm is performed. The result is shown in Fig. 11. In the green circle of Fig. 12, one can figure that the floor pillar doesn't converge well as prediction is applied.

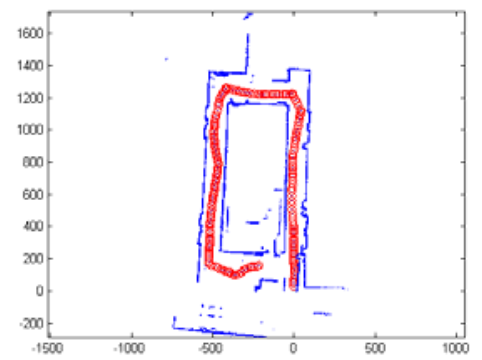

Fig. 12. Result of proposed scheme without prediction

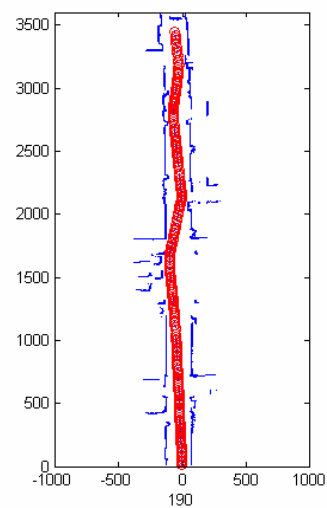

Fig. 13. Result for long corridor environment 
In another experiment, a long corridor environment is used to test the accumulation error. The result is shown in Fig. 13. The total distance of the corridor is $35 \mathrm{~m}$, and 72 scanned data is captured to construct the environment.

The last experiment is the largest scale, the whole environment of office. 450 data are used to construct the map. The result is shown in Fig. 14.

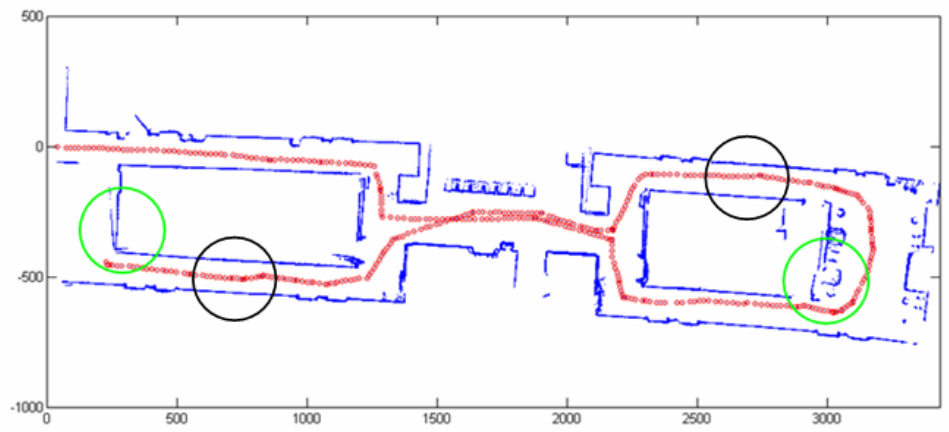

Fig. 14. Result for large scale indoor environment

In the green circle from above figure, the affect of accumulation error is apparent. This is caused by the poor point feature in the parallel wall scanned in the corridor (the black circle shown in Fig. 13.) To overcome this drawback, global optimization algorithms should be applied in the future.

\section{Conclusion}

In this paper, we proposed a scheme which integrates distance transform and the concepts of prediction into map mapping task. And the mapping problem is transform into a optimization problem. The experiment shows it could be continued in the surrounding environment. Even the maps have 450 maps to composition. The proposed scheme still gets efficiency to complete the SLAM task. However, there are some problems in the result and need to be overcome in the future. Although the integrated error has been decreased by prediction algorithm, but for a large scale environment it still exist. Global map optimizations algorithms need to be develop to overcome this drawback.

\section{References}

1. Whyte, H.D., Bailey, T.: Simultaneous Localization and Mapping: Part I. IEEE Robot. Autom. Mag. 13(2), 99-110 (2006)

2. Dissanayake, M., et al.: A Solution to the Simultaneous Localization and Map Building (SLAM) Problem. IEEE Trans. Robotics, Automation 17(3), 229-241 (2001)

3. Robert, O., Kotaro, H.C.: A Comparison of SLAM Implementations for Indoor Mobile Robots. In: IEEE/RSJ International Conference on Intelligent Robots and Systems, San Diego, pp. 1479-1484 (2007) 
4. Martinelli, A., et al.: A Relative Map Approach to SLAM Based on Shift and Rotation Invariants. Robotics and Autonomous Systems 55(1), 50-61 (2007)

5. Gutmann, J.-S.: Robuste Navigation autonomer mobiler Systeme. PhD thesis, AlbertLudwigs-Universität Freiburg (2000)

6. Hähnel, D., Burgard, W., Fox, D., Thrun, S.: An efficient FastSLAM algorithm for generating maps of large-scale cyclic environments from raw laser range measurements. In: IROS 2003, vol. 1, pp. 206-211. IEEE, Los Alamitos (2003)

7. Rongchuan, S., Shugen, M., Bin, L., Yuechao, W.: Simultaneous Localization and Sampled Environment Mapping. In: 48th IEEE Conference on Decision and Control and 28th Chinese Control Conference, Shanghai, P.R. China, pp. 6684-6689 (2009)

8. Besl, P.J., McKay, N.D.: A method for registration of 3D shapes. IEEE Transactions on Pattern Analysis and Machine Intelligence 14(2), 239-256 (1992)

9. Lu, F.: Shape Registration Using Optimization for Mobile Robot Navigation. PhD thesis, University of Toronto (1995)

10. Lu, F., Milios, E.: Robot pose estimation in unknown environments by matching 2D range scans. J. of Intelligent and Robotic Systems 20, 249-275 (1997)

11. Nguyen, V., Gächter, S., Martinelli, A., Tomatis, N., Siegwart, R.: A comparison of line extraction algorithms using $2 \mathrm{D}$ range data for indoor mobile robotics. Autonomous Robots 23(2), 97-111 (2007)

12. McFetridge, L., Ibrahim, M.Y.: A new methodology of mobile robot navigation: The agoraphilic algorithm. In: Robotics and Computer-Integrated Manufacturing, pp. 545-551. Elsevier Press, Amsterdam (2009)

13. Castej, C., Blanco, D., Boada, B.L., Moreno, L.: Voronoi extraction of free-way areas in cluttered environments. In: The 11th international conference on Advanced Robotics. Coimbra, Portugarl (2003)

14. Nidhi, K., Dave, F., Anthony, S.: Incremental Reconstruction of Generalized Voronoi Diagrams on Grids. In: International Conference on Intelligent Autonomous Systems(IAS), IOS Press, Amsterdam (2006)

15. Minguez, J.: The Obstacle-Restriction Method (ORM) for Robot Obstacle Avoidance in Difficult Environments. In: Intelligent Robots and Systems, pp. 3706-3712. IEEE press, Los Alamitos (2005)

16. Lars, H., Hanna, S.: An evaluation of measures for quantifying map information. ISPRS Journal of Photogrammetry and Remote Sensing (2007)

17. Felix, W., Frederic, M., Joaquin, S., Howie, C., Stephen, T., George, K.: Topological SLAM using Neighbourhood Information of Places. In: IEEE/RSJ International Conference on Intelligent Robots and Systems, pp. 4937-4942. IEEE press, St. Louis (2009) 